\title{
La noción de dignidad humana en la Corte Constitucional Colombiana \\ Una mirada alternativa desde Martha Nussbaum
}

\section{Resumen}

Andrés Sandoval Sarrias*

En este artículo me propongo hacer un acercamiento al concepto de dignidad humana desde su apropiación en la Constitución Política de Colombia de 1991 y las interpretaciones que la Corte Constitucional ha hecho en sus sentencias. Sostendré que el fundamento del concepto de dignidad que interpreta la Corte tiene en sus raíces el kantismo y estableceré un diálogo entre esta interpretación que Nussbaum hace de la dignidad humana. Argumentaré que: a) la comprensión que hace la Corte del concepto de dignidad es insuficiente para casos complejos. b) Es posible establecer una idea de la dignidad humana desde una fuente alternativa al kantismo si defendemos un esencialismo al modo aristotélico. c) Por lo tanto, la propuesta altenativa presentada por Nussbaum puede ser una opción más apropiada para algunas sentencias tutelares de la Corte Constitucional Colombiana

Palabras clave: Dignidad, Esencialismo, Constitución, Capacidades Básicas, Corte.

\section{The notion of human dignity in the Colombian Constitutional Court An alternative look from Martha Mussbaum}

\begin{abstract}
In this article I will realize an approach to human dignity concept from the appropriation that Political Constitution of Colombia in 1991 and the interpretations done by Constitutional Court in its Sentences. I will hold the Court's interpretation of the concept dignity has its roots in the Kant's understanding of dignity; then I will stablish a dialogue between Court's interpretation of dignity in Kant and the understanding of dignity in Nussbaum's conception. I will argue: $\boldsymbol{a}$ ) the understanding that makes the Court of the concept of dignity is insufficient for complex cases. b) It is possible to establish a sense of human dignity from an alternative source Kantianism if we defend the Aristotelian essentialism mode. $c$ ) Thus the alternative

*Universidad Libre, Cali-Colombia. asandoval.s@gmail.com Páginas: 15-39
\end{abstract}


interpretation suggested by Nussbaum may be more appropriate for the tutelary veredicts of the Colombian Constitutional Court.

Key words: Dignity, Essentialism, Constitution, Basis Capabilities, Court.

Sumario: I. Dignidad humana en contexto. II. Apropiación de la dignidad en las Constituciones de América Latina. III. La apropiación del concepto de dignidad en la Corte Constitucional. IV. El enfoque de las capacidades y la dignidad. V. Una propuesta esencialista: las funciones humanas básicas. VI. Capacidades básicas y dignidad. Bibliografía

\section{Dignidad humana en contexto}

La dignidad como percepción universalizada de una cualidad humana inalienable e igual para todos los hombres es ante todo una idea moderna. Si bien se puede rastrear algunas nociones primitivas del concepto en la filosofía antigua y medieval, éstas están asociadas al valor y el honor o vinculadas con una filiación que revestía a unos pocos con una distinción particular. "En efecto, tanto en la antigüedad, la Edad-Media, el Renacimiento, etc. el valor del individuo derivaba de su filiación, origen, posición social, u otros cargos políticos. En resumen, los individuos nacían con dignidades distintas y desiguales" (Pele, 2004:10). Podemos entonces decir que si bien hay una concepción pre-moderna de la dignidad humana, ésta hacía referencia a una valía adquirida por filiación o título honorífico cuyo valor estaba en que era otorgado a unos pocos.

Por otro lado, la concepción moderna de dignidad forma parte de la ética pública y se vincula a la naturaleza humana de manera intrínseca y sobre todo igualitaria en su contenido. Como paradigma político y jurídico de la modernidad, está vinculada a una ética pública cimentada en cuatro grandes valores: la libertad, la igualdad, la solidaridad y la seguridad jurídica.

"La idea de dignidad humana,-subraya Peces-Barba- para su realización

a través de la vida social, (es) inseparable de la condición humana, se 
plasma en esos cuatro valores, cuyo núcleo esencial lo ocupa la libertad, matizada y perfilada por la igualdad y la solidaridad, en un contexto de seguridad jurídica”. (Peces-Barba, 2007:157).

Y aunque haya pluralidad en las posturas ideológicas sobre la dignidad, el establecimiento de los Derechos Humanos que siguió a la postguerra nos heredó la idea de una universalización y unificación de la dignidad que se materializó en la Declaración de los Derechos Humanos de 1948. Como puede evidenciarse, esta concepción moderna de la dignidad dista mucho de sus alcances en las épocas que la precedieron.

La dignidad, en esta línea, representa una expresión del respeto y valor que debe otorgarse al ser humano en virtud de su condición humana, cimenta además las bases de la convivencia y se articula estrechamente a la noción de Derechos Humanos. Además, la dignidad humana se constituye como el sustrato y el punto de partida de todos los derechos humanos que se diferencian a partir de ella, y a la vez actúa como un punto de vista que da perspectiva a los diferentes derechos humanos lo que permite entenderlos e interpretarlos (Cfr.Stix-Hackl, 2004: Párrafo 76).

Para Kant, la dignidad es el valor fundamental que no se puede negociar al ser el fundamento de los demás valores que constituyen la vida humana. Para el pensador alemán "la dignidad es el atributo de un ser racional que no obedece a ninguna otra ley que la que él mismo se da". Es así que "la autonomía es el fundamento de la dignidad de la naturaleza humana o de toda naturaleza racional" que debe ser respetada y cuyo peso moral se rige por el imperativo categórico "obra de tal modo que te relaciones con la humanidad, tanto en tu persona como en la de cualquier otro, siempre como un fin y no como un medio". La dignidad entonces constituye la razón fundamental por la que la vida humana se equipara en valor, obligando moralmente a los hombres a consolidar las bases del autorrespeto y el respeto a los demás.

En la Fundamentación de la metafísica de las costumbres, Kant afirma que la idea de dignidad humana es producto de su autonomía y autodeterminación 
moral que hace de la persona humana algo más que lo esencialmente físico. Ese valor interior que expresa la condición moral y que Kant llama dignidad está sujeto al respeto en igual condición para todos y es de allí que deriva el imperativo categórico que prohíbe instrumentalizar a cualquier persona. Kant explica la dignidad como un hecho autoevidente a la razón. Esto significa que cualquiera está en la capacidad de reconocerla y por tanto de respetar la suya y la de los demás, o lo que es lo mismo, que la dignidad es autoevidente a la razón humana (Restrepo, 2011:8-9). Esta es quizás la característica fundamental que nos ha dejado la modernidad y que, para bien o para mal, permeó todas las esferas de la sociedad que de una u otra manera necesita de un concepto de esta envergadura para fundamentar sus luchas sociales o sus barbaries. El derecho, por supuesto, no ha sido ajeno a este entendimiento.

\section{Apropiación de la dignidad en las Constituciones de América Latina}

Tras la primera guerra mundial que dejo millones de muertes y barbarie, se creía que un suceso de tal magnitud no podía repetirse nuevamente. Sin embargo, con la escalada expansionista de la Alemania Nazi, el mundo asistió a el peor capítulo de su historia reciente descubriendo que la maldad humana no tenía límite conocido y que el extermino bajo el argumento de superioridad racial era aún vigente para muchos. Las nuevas formas de guerra, las bombas atómicas y el extermino al que fueron sometidas culturas enteras, dejaron un sinsabor de retroceso en el progreso moral que persistió aún terminada la guerra. Por ello, en el año de 1945 cuando la reconstrucción mundial apenas comenzaba, cincuenta naciones se reunieron en San Francisco con la esperanza y la firme intención de establecer parámetros que no sólo regularan la guerra sino que establecieran criterios universales de protección de la vida humana que llegara a todos los rincones de la tierra.

El producto de esta reunión fue la creación de la ONU (Organización de las Naciones Unidas) con la intención de proteger y promover la paz en las generaciones venideras. Con la tarea de trazar un sendero de respeto a la vida humana y promover el entendimiento entre las naciones, en el año 1948 la comisión de la ONU acogió Declaración universal de los derechos humanos 
como carta tutelar de ordenamiento que tuvo como consigna la idea de que todo ser humano, en tanto humano, nace libre e igual en derechos. Así, bajo la mirada del mundo, se redactó una nueva Carta Magna con un total de 30 artículos que se convirtieron en la bitácora mundial para las constituciones políticas modernas así como se estableció la autoridad para intervenir en naciones donde estos derechos no se respetaran.

La primera nación en apropiar estos principios fue la renaciente Alemania en su Ley Fundamental de Bonn, la primera Constitución Europea en poner la dignidad a la base de la democracia en 1949. El primer artículo es enfático en señalar que "La dignidad humana es intangible. Respetarla y protegerla es obligación de todo poder público". "El pueblo alemán, por ello, reconoce los derechos humanos inviolables e inalienables como fundamento de toda comunidad humana, de la paz y de la justicia en el mundo". Con este primer paso, rápidamente las democracias modernas siguieron este modelo y América Latina no fue la excepción.

La dignidad pasó a jugar un papel muy importante para la justificación de la protesta, el derecho al trabajo y sobre todo el papel de la libertad y la igualdad frente a la ley que sólo puede ser amparada bajo la idea de una dignidad inherente a la persona humana. Mientras en Constituciones como la de República Dominicana la dignidad apenas si se menciona y otras como la uruguaya y la argentina ni siquiera se menciona explícitamente, otras se han apropiado del concepto con mayor rigidez. En países con pasados dictatoriales fuertes como Paraguay y Cuba, inclusive Venezuela, la dignidad se usa constantemente como justificación de los derechos y los deberes políticos de los ciudadanos, en el resto de las constituciones latinoamericanas se tiene un abordaje del concepto particularmente en tres ámbitos: el respeto a la vida, el trabajo y la libertad en contraposición a la esclavitud.

\section{La apropiación del concepto de dignidad en la Corte Constitucional}

En los artículos 1, 42, 53 y 70 de la Constitución Política de Colombia de 1991 se establece que la dignidad humana es un elemento base para la protección de los derechos fundamentales y la promoción de la libertad, la 
justicia y la igualdad. La Corte Constitucional, en el ejercicio de sus facultades, se encarga de determinar los lineamientos de la naturaleza práctica formal que se invoca en estos artículos con el propósito de establecer una jurisprudencia que permita a los ciudadanos hacer uso de sus derechos y exigir restauración cuando éstos son violados.

En el caso de la dignidad, la sentencia T-881 de 2002 sintetiza muy bien el entendimiento de la naturaleza de la dignidad humana en la jurisprudencia al interpretarla de dos maneras:

A partir de su objeto concreto de protección y a partir de su funcionalidad normativa. Al tener como punto de vista el objeto de protección del enunciado normativo "dignidad humana", la Sala ha identificado a lo largo de la jurisprudencia de la Corte, tres lineamientos claros y diferenciables: (i) La dignidad humana entendida como autonomía o como posibilidad de diseñar un plan vital y de determinarse según sus características (vivir como quiera). (ii) La dignidad humana entendida como ciertas condiciones materiales concretas de existencia (vivir bien). Y (iii) la dignidad humana entendida como intangibilidad de los bienes no patrimoniales, integridad física e integridad moral (vivir sin humillaciones). De otro lado al tener como punto de vista la funcionalidad, del enunciado normativo "dignidad humana", la Sala ha identificado tres lineamientos: (i) la dignidad humana entendida como principio fundante del ordenamiento jurídico y por tanto del Estado, y en este sentido la dignidad como valor. (ii) La dignidad humana entendida como principio constitucional. Y (iii) la dignidad humana entendida como derecho fundamental autónomo. (Sentencia T-881)

Asimismo, al hacer alusión al contenido material de la dignidad humana, ésta se vincula con tres ámbitos exclusivos de la persona en la jurisprudencia colombiana: "su autonomía individual, unas condiciones de vida cualificadas y la intangibilidad del cuerpo y del espíritu. Estos tres ámbitos de protección integran, entendidos en su conjunto, el objeto protegido por las normas constitucionales desarrolladas a partir de los enunciados normativos sobre "dignidad". Considera la Corte que ampliar el contenido de la dignidad humana, 
con tal de pasar de una concepción naturalista o esencialista de la misma en el sentido de estar referida a ciertas condiciones intrínsecas del ser humano, a una concepción normativista o funcionalista en el sentido de completar los contenidos de aquella, con los propios de la dimensión social de la persona humana, resulta de especial importancia, al menos por tres razones: primero, porque permite racionalizar el manejo normativo de la dignidad humana, segundo, porque lo presenta más armónico con el contenido axiológico de la Constitución de 1991, y tercero, porque abre la posibilidad de concretar con mayor claridad los mandatos de la Constitución. Los ámbitos de protección de la dignidad humana, deberán apreciarse no como contenidos abstractos de un referente natural, sino como contenidos concretos, en relación con las circunstancias en las cuales el ser humano se desarrolla ordinariamente" (Sentencia T-881).

No obstante, la naturaleza de la dignidad humana no parece ser del todo un concepto jurídicamente "claro y diferenciable" como sugiere la sentencia. (i) la idea de dignidad humana como la autonomía para realizar un plan de vida que se ajuste a "vivir como quiera" no es eficaz definiendo las capacidades y las oportunidades reales que tienen las personas para vivir una vida que consideran deseable. Así, se puede argumentar que alguien es autónomo para elegir un plan de vida cuando en realidad las cadenas de pobreza le están quitando su capacidad real de elegir. (ii) la dignidad entendida como "ciertas condiciones materiales" para "vivir bien" es un abanico tan amplio y tan variable que conceptualmente no aporta mucho. Pasa por encima de la diferenciación cultural, los fines propios y pasa por alto sutiles diferenciaciones que enmarcan la vida humana. (iii) más compleja aún es la idea de dignidad como "vivir sin humillaciones".

La humillación, al igual que otras emociones como la ira, el malestar y el resentimiento están sujetos a los sentimientos morales de cada persona, sus concepciones de vida y su autorrespeto. Así, esta apreciación tan ambigua ha dado lugar a tutelas en las que una persona padeciendo dolor que siente le reduce su dignidad, tutela su derecho a una vida y muerte digna pero la Corte falla en su contra argumentando la protección a su dignidad (véase Sentencia 
T-970/14). ¿De qué estamos hablando cuando hablamos de dignidad? ¿Cuál es su sistema de medidas y cuáles son los criterios y procedimientos fijados en la Corte para proferir un fallo en torno a la dignidad? ¿Quién tiene la autoridad moral para clasificarla? ¿Puede proveerse una aproximación más certera al concepto de dignidad que apoye más firmemente el derecho jurisprudencial colombiano? Fundamentalmente ¿Podemos establecer un principio rector de interpretación de la dignidad tal es que sea igualmente aplicable en todos los fallos en donde se requiera?

La Corte, en el ejercicio de sus funciones, asume como verdadera la idea de que el ser humano posee una dignidad connatural a todo ser humano y para cuya protección trabaja el organismo jurídico. Al menos así lo deja entrever algunas sentencias:

La dignidad, como es sabido, equivale al merecimiento de un trato especial que tiene toda persona por el hecho de ser tal. Equivale, sin más, la facultad que tiene toda persona de exigir de los demás un trato acorde con su condición humana. (Sentencia T-395, T-576)

El respeto de la dignidad humana, esto es, del valor intrínseco del ser humano, derivado de sus atributos específicos como son la voluntad y la razón, es uno de los principios fundamentales del Estado Social de Derecho colombiano. (Sentencia C-397)

La influencia kantiana es directamente relacionada en algunos fallos

...la dignidad de la persona debe ser considerada, primariamente, como aquel valor constitucional que busca proteger al individuo en tanto ser racional y autónomo, capaz de adoptar las decisiones necesarias para dar sentido a su existencia y desarrollar plenamente su personalidad y, de conformidad con ello, determinar sus acciones sin coacciones ajenas de ninguna índole. El objeto fundamental del principio de la dignidad de la persona es, entonces, la protección del individuo como fin en sí mismo, el individuo como universo único e irrepetible con capacidad para darse sus propias leyes morales, las cuales, en razón de que los otros son, también, fines en sí mismos, deben ser 
compatibilizadas con las de las otras personas. El principio de la dignidad humana protege como diría Kant al individuo autolegislador en un reino de fines. (Sentencia T-472)

Así, parece entonces que la Corte ha validado dentro de sus sentencias los principios de la dignidad que son comunes a una línea de interpretación del kantismo, la idea de dignidad como autoevidente a la razón. Hay que decir que para bien o para mal, esta noción ha operado con éxito en la legislación colombiana. Sin embargo, lo que aquí me propongo es hacer un acercamiento a una nueva corriente contemporánea que busca hacerse un lugar en las interpretaciones de la dignidad que parecen tener algo que decirnos en la esfera del derecho. Quiero pasar ahora a presentar el enfoque de las capacidades y la defensa del esencialismo aristotélico con el propósito de presentar una visión alternativa de entendimiento de la dignidad, ya no simplemente como un elemento abstracto sino viablemente y medible. En la parte final realizaré un análisis final con el fin de mostrar en qué puntos de interpretaciones de Nussbaum puede resultar en una valiosa herramienta para las sentencias que la Corte debe proferir en algunos casos límite. Argumentaré, que el mejor aporte de Nussbaum es el carácter práctico y "medible" que alcanza la dignidad dentro del enfoque de las capacidades.

\section{El enfoque de las capacidades y la dignidad}

El enfoque de las capacidades nació en el seno de la teoría de Amartya Sen como respuesta a las carencias de los modelos económicos y del desarrollo que invisibilizaban las injusticias que se cometen al interior de una nación. El enfoque es una nueva propuesta para evaluar la justicia a partir de un examen de las posibilidades reales que tienen las personas para elegir una vida que consideran deseable y se miden en términos de capacidades, y éstas a su vez se ven reflejas en los funcionamientos alcanzados. Un funcionamiento puede ser el estar bien nutrido o saber leer, la capacidad se medirá en la libertad real que la persona tuvo de adquirir esos funcionamientos. El enfoque entonces "se interesa principalmente en la identificación de los objetosvalor, y considera al espacio evaluativo en términos de funcionamientos y 
capacidades para funcionar.'(Nussbaum \& Sen: 1993:57). Esta propuesta alternativa de evaluación de la justicia planteada por Sen es compartida con Martha Nussbaum quien pretende darle un giro al enfoque a fin de ajustarlo de manera más práctica.

En los desarrollos posteriores que Nussbaum realiza del enfoque, ella va a distinguir una lista de capacidades que considera básicas para establecer un mínimo de calidad de vida con arreglo a la dignidad. Según Nussbaum, estas capacidades básicas son intercambiables con los derechos fundamentales, de allí que sea necesario evaluar la viabilidad de esa aspiración. En este apartado entonces voy a presentar el pensamiento de Nussbaum comenzando por su defensa del esencialismo como elemento de justificación para las capacidades básicas y por lo tanto de los derechos.

Nussbaum tiene claro que para defender la idea de una dignidad humana, capacidades básicas y sobre todo los derechos como capacidades, tendrá que defender una visión de la vida humana de alcance universalista. Reconociendo que el esencialismo tiene que afrontar múltiples críticas, se da a esta tarea en su artículo Human Functioning and Social Justice.

\section{Una propuesta esencialista: las funciones humanas básicas ${ }^{1}$}

En términos más generales, Nussbaum considera que la defensa de una visión esencialista de la persona humana, esto es, la defensa de que los seres humanos poseen características comunes tales es que podemos determinar-al menos en un nivel mínimo- sus necesidades, deberá afrontar al menos tres objeciones principales. La primera será el descuido a las diferencias culturales e históricas. La humanidad se entiende desde diferentes perspectivas y cualquier intento por simplificarla y universal sus componentes fundamentales termina por atentar contra nociones culturales o históricas importantes para una comunidad particular. Todo intento por construir una lista de elementos esenciales termina por incluir ciertos modos de comprender la vida y excluir otros que en principio son igualmente válidos. Se necesitaría un consenso universal y tal coso no es fácticamente posible.

${ }^{1}$ ANESSENTIALISTPROPOSAL: THE BASIC HUMAN FUNCTIONS. 
La segunda es el denominado descuido a la autonomía. Esta crítica viene principalmente de los liberales que pese a que estarían acuerdo en ser esencialistas en la libertad y la autonomía, presentarían granes reservas a la idea de determinar de antemano los principios fundamentales que componen la vida humana pues con ellos se atentaría contra el derecho de elegir un plan de vida donde cada quién decida lo que considera bueno y deseable. Por lo tanto, toda teoría política debe negarse a sí misma una teoría determinada del ser humano y del bien humano.

La tercera crítica es en lo referente a la aplicación de prejuicios. Al optar por una concepción normativa de lo que constituye el ser humano, esto tendrá consecuencias morales y políticas que facilitarán la exclusión de aquellos que no tengan suficientes fuerza participativa como para defender su propia forma de vida. El mismo Aristóteles consideraba que algunas personas tales como las mujeres y los esclavos no cumplían los lineamientos que constituían su teoría esencialista de la vida humana. Por lo tanto, toda concepción esencialista de la vida humana acarrea consecuencias políticas y morales de exclusión para aquellos que no gozan de suficiente reconocimiento como para cumplir esos estándares. Esto sin duda da lugar daría lugar a muchas injusticias y conflictos.

El propósito de Nussbaum es identificar un grupo de funciones básicas especialmente importantes para la vida humana como respuesta, en gran medida, al relativismo. Esto nos permitirá ver el tratamiento que las instituciones sociales hacen de ellas y por consiguiente evaluar su promoción o violación. La llama "teoría vaga y gruesa del bien"2, una lista con carácter normativo. No metafísica sino histórica, no religiosa y fundada en dos hechos: que siempre a los otros como humanos a pesar de las divisiones de tiempo y lugar. Dado que difícilmente estaremos inseguros de si estamos o no tratando

\footnotetext{
${ }^{2}$ Nussbaum habla de una "thick vague theory of the good" para hacer un contraste una delgada teoría del bien que presenta Rawls y que implica reducir los bienes primarios que serán usados por las personas en la posición original, un grupo de medios de aplicación universal que juegan un papel relevante en cualquier concepción del bien humano. En contraste la teoría gruesa se basa en la teoría de los fines aristotélica sobre el contenido general de las formas de vida humana. (Cf. Nussbaum: 1992:214-215).
} 
con humanos, la TVGB intenta describir las bases de ese reconocimiento humano apelando a aquellos elementos que conforman el carácter y la forma de la vida humana donde quiere que ésta se dé. El segundo es establecer un consenso general de aquellos caracteres que, en su ausencia en la vida humana, nos permiten decir de una vida humana que ya no lo es. Estos apelan a los rasgos más fundamentales de nuestra humanidad común sin los cuales se percibiría que ya no estamos ante una presencia humana (Cf. Nussbaum: 1992:215).

Establece unos "niveles" aproximativos de su teoría esencialista que configuran la vida humana. La primera es la figura de la forma se vida humana (Cf. Nussbaum: 1992:216). A juicio de Nussbaum esta vaga teoría está compuesta por 10 elementos: mortalidad, la figura del cuerpo humano, la capacidad para sentir placer y dolor, una serie de facultades cognitivas tales como la percepción, la imaginación y el pensamiento, un desarrollo infantil temprano, razón práctica, capacidad para socializar con otros seres humanos así como capacidad y afinidad para con otras especies y la naturaleza, finalmente, la lista se cierra la idea de que el ser humano tiene sentido del humor y lúdico y su existencia es en separación con otros seres (Cf. Nussbaum: 1992:216220). Voy a profundizar en estos aspectos un poco más.

La mortalidad es la primera y fundamental característica de un ser humano. Morir es el destino inevitable de cualquier ser humano y si estuviésemos frente a un ser con la capacidad de extender al infinito su existencia, entonces muy seguramente cuestionaríamos su humanidad. Sin embargo, la concepción de mortalidad de Nussbaum no se limita a esta premisa. Según ella, este caracteriza involucra la conciencia de una muerte cercana al llegar la vejez, un rechazo a la muerte pero a la vez un aprecio en circunstancias en las que se percibe preferible a ciertos estados de vida. Así, cuestionaríamos una forma de vida humana si en ella no estuviera presente la inminencia de la muerte o una falta de aversión a ella.

Respecto de la concepción de un cuerpo humano, Nussbaum lo cataloga como cierto recipiente en el que nuestra humanidad es contenida; así, 
puede haber una profunda variación en la forma que éste tenga pero una universalización de su propósito y contenido. Pero aun así esta mera concepción no nos advierte suficiente sobre la "humanidad" contenida en ese cuerpo, es por eso que nuestra autora señala algunos aspectos adicionales que componen ese cuerpo humano. En primer lugar, todo cuerpo humanos tiene la necesidad de comida y bebida, estos, experimentan sed y hambre; todos los seres humanos tienen la necesidad de alimentarse e hidratarse independiente de su deseo de hacerlo tal es que dudaríamos de la humanidad de alguien que careciera de esta necesidad. Así mismo, todo ser humano parece tener la necesidad de abrigo y hogar. Un lugar común de toda concepción histórica o mitológica de humanidad está atravesado por la necesidad de cubrir el cuerpo de la inclemencia de los elementos y buscar lugares para sobreguarda la protección de su cuerpo. Adicionalmente, una característica "corporal” del hombre es su apetito sexual; sin demandar la urgencia de la alimentación, la los deseos sexuales son constitutivos de la persona humana y tiene vital importancia para reconocer los otros como seres humanos. Finalmente, la movilidad propia de los seres humano constituye una rasgo importante de su corporeidad; esto implica no sólo la habilidad de movernos de una lugar a otro haciendo uso de nuestro cuerpo o de herramientas que hayamos construido sino también la necesidad de estar en constante movimiento acompañado de un rechazo a la privación de la movilidad.

La siguiente característica es la capacidad de sentir placer y dolor. Se considera que toda forma de vida humana está sujeta a un conjunto de sensaciones que catalogamos como placenteras o dolorosas y que además tendemos a generar un rechazo hacia el dolor de manera natural. Esto es, el rechazo al dolor como un rasgo primitivo de nuestra humanidad y no como algo que se aprendió o incorporó a la vida humana. Así, una sociedad donde no existiera el miedo al dolor, puntualiza Nussbaum, sería juzgada como sobrepasado los límites de lo humano.

La facultad cognitiva, que está acompañada de la percepción, la imaginación y el pensamiento, se presenta como un lugar común en toda concepción humana. Esto no implica una estandarización en los contenidos de 
estas percepciones y pensamientos sino una capacidad de llegar a comprender hechos que involucran su existencia o que lo afectas, aunque esta capacidad varíe en forma y amplitud en casa ser humano. De cualquier manera, Nussbaum considera que una sociedad, pueblo o tribu que cuyos integrantes carecieran de estos elementos, pondría en cuestionamiento nuestra capacidad para clasificarlos como propiamente humanos.

El desarrollo infantil temprano hace referencia a la linealidad de la vida humana. Empezamos la vida como bebés y padecemos de total dependencia de terceros durante una parte de nuestra vida. Este desarrollo temprano es condición de todo ser humano y está acompañado de la formación de deseos y emociones como el amor, el dolor y el miedo. Nuestro desarrollo infantil además configura parte de nuestra personalidad y acumula una serie de experiencias fundamentales para el desarrollo posterior y la adquisición de nuevas capacidades sociales y cognitivas. Así, si llegase a existir una tribu o pueblo donde sus integrantes no hayan pasado por este desarrollo infantil, seguramente tendríamos serias consideraciones para catalogarlos como de nuestra misma especie.

La razón práctica es la siguiente característica que a juicio de Nussbaum constituye la vida humana. Esto implica que todo ser humano en algún punto de su existencia desea planificar y dirigir sus propias vidas y desarrollan una jerarquía de valores que configuran una concepción del bien y de la vida. Una razón práctica implica la capacidad de idearse un plan de vida e intentar seguirlo haciendo uso de otras capacidades como las antes mencionadas. Así, si encontráramos alguien que careciera por completo de esta característica, seguramente podríamos decir que su humanidad no está plenamente desarrolla y por lo tanto tampoco se consideraría plenamente humano.

Conectada a estas últimas, la sociabilidad constituye a juicio de Nussbaum una característica central de la persona humana. Todo ser humano tiene la capacidad de relacionarse con otros seres humanos y de desarrollar sentimientos de preocupación por otros seres humanos. Esto implica, siguiendo los principios aristotélicos, que nos definimos al menos por dos 
clases de filiación: en las relaciones familiares que involucran una esfera íntima, y las relaciones sociales y cívicas por las que establecemos lazos sociales. En definitiva, afirma Nussbaum, vivimos para los demás y con ellos, y consideraríamos poco digna una vida una vida donde esa capacidad de relacionarse se viera truncada.

Así mismo, derivaba de esa capacidad para socializar con los seres humanos, la filósofa considera que todo ser humano tiene la capacidad de establecer vínculos afectivos con otros seres vivientes y con la naturaleza; el ser humano tiene la capacidad de reconocer su dependencia de otras especies y de la naturaleza, admirar su belleza y comprender sus limitaciones y responsabilidades para con otras especies pese a que hayan divergencias en la forma correcta en que debemos tratarlos. De nuevo, si careciéramos de una incapacidad total para establecer vínculos con otros seres vivos o con la naturaleza en general, probablemente diríamos que hay un aspecto central de la vida humana que se encuentra cercenado afectando profundamente nuestra humanidad.

La capacidad de sentir humos y adquirir un sentido lúdico aparece también como fundamental en la configuración dela persona humana. La risa se constituye en un lenguaje universal. El reposo, el recreo y el juego son partes importantes de la constitución de una cultura y transitivamente importantes para la configuración adecuada de una vida humana. Carecer de esta capacidad para reír, jugar o divertirse alteraría profundamente la capacidad de reconocernos como humanos y de reconocer a otros como tal, dejando en serios aprietos la clasificación de una vida de este tipo como verdaderamente humana. Además, puntualiza Nussbaum, viviríamos una vida muy extraña y espantosa.

Finalmente, la lista se cierra con la separación o separabilidad de cada individuo como único, irrepetible, y ocupando un lugar singular que no es ocupado nunca en simultáneo por alguien más. Si bien la sociabilidad es un aspecto fundamental del ser humano, lo es también su unicidad, es decir, nadie puede sentir por mí, vivir conmigo en la misma esfera de tiempo y espacio que 
yo ocupo y que comienza de manera individual desde el nacimiento hasta la muerte. Si estamos ante la presencia de seres humanos diversos, no tenemos problemas para identificar su singularidad constituida por el lugar que ocupa, su contexto, historia, experiencias y lazos que, sin importar el grado y la extensión, son comunes como experiencia de cada ser humano. En suma, cada ser humana debe tener, y además desea, alguna forma de individualidad que le permita distinguirse de los demás y configurar un especio propio y una historia propia.

Esta extensa lista constituye el primer nivel de entendimiento de la forma de vida humana marcada por sus límites y sus capacidades. De cualquiera de estos elementos podríamos decir que en su ausencia la vida humana sería insuficiente y sobre todo no sería una buen $a^{3}$, esto es, se carecería de un piso básico para establecer una concepción mínima del bien. Ahora bien, este primer esfuerzo sería inútil si no se acompaña del establecimiento de unos umbrales básicos que constituyeran la capacidad para funcionar que cada persona requiere. Ese umbral, sería el límite último para determinar cuándo estamos ante una vida humana verdadera y cuándo esa idea se tono borrosa. Este umbral se establece no como plan último de la configuración de una vida buena sino como el establecimiento de límites mínimos para la construcción de un límite inferior, así, "[E]l umbral último será el que más nos preocupe a la hora de volverla política pública, debido a que no queremos que las sociedades hagan a sus ciudadanos capaces del mínimo indispensable" (Nussbaum: 1992:221 $)^{4}$. Esto implica que en un nivel superior, ya no se intenta configurar los aspectos generales de la vida humana sino entablar la búsqueda de unos principios de vida buena con arreglo a esa naturaleza ya definida por esos límites y capacidades.

Habiendo configurado este primer nivel, Nussbaum da un paso adelante para establecer una nueva lista (derivada como se puede esperar de la

\footnotetext{
${ }^{3}$ Nussbaum insiste en su vaga y gruesa teoría apunta no sólo a los componentes esenciales de la vida humana sino también al establecimiento de una vida con arreglo a fines de dignificación, es decir, a llevar una vida buena que valga la pena ser vivida y que por lo tanto esté en concordancia con la dignidad.

${ }^{4}$ The latter thresholds the one that will eventually concern us most when we turn to public policy, for we do not want societies to make their citizens capable of the bare minimum.
} 
primera), ya no para delimitar la vida humana en general sino para establecer las capacidades que se deberán promover dentro de una comunidad política que se preocupe por el bienestar y la dignidad de sus ciudadanos. Este segundo umbral se elabora en términos de la capacidad para funcionar que a juicio de la autora debería establecer las bases de la planificación pública en beneficio de las personas. En esa medida, este nuevo umbral y esta nueva lista, se configura como capacidades funcionales básicas de toda persona humana y de allí es que se hereda el concepto de capacidades básicas.

Como la anterior lista, ésta se compone de 10 capacidades de la siguiente forma:

1. Ser capaz de vivir una vida hasta el final extendiéndola tanto como sea posible; evitar la muerte prematura o tener una vida que se vea tan reducida en sus posibilidades que no valga la pena ser vivida.

2. Ser capaz de gozar de buena salud, estar adecuadamente nutrido y gozar de un techo adecuado. Así mismo tener la oportunidad para satisfacer los deseos sexuales y gozar de la movilidad para desplazarse de un lugar a otro.

3. Ser capaz de evitar dolor innecesario y no beneficioso, así como gozar de experiencias placenteras.

4. Ser capaz de usar los cinco sentidos, ser capaz de imaginar, pensar y razonar.

5. Ser capaz de establecer vínculos con personas y cosas fuera de nosotros mismos, amar y cuidar a aquellos que cuidan de nosotros y nos aman, sentir pena en su ausencia y en general amar, padecer y sentir nostalgia y gratitud.

6. Ser capaz de formar una concepción del bien y comprometerse en una reflexión crítica a cerca de la planeación de nuestra propia vida.

7. Ser capaz de vivir con y para los otros, reconocer y mostrar preocupación por otros seres humanos, comprometerse en varias formas de interacción familiar o social. 
8. Ser capaz de vivir con la preocupación y relación con los animales, plantas y el mundo de la naturaleza.

9. Ser capaz de reír, jugar, y disfrutar de actividades recreativas.

10. Ser capaz de vivir la vida propia y no la de alguien más. Poder vivir la propia vida en un entorno y contexto propio (Cfr. Nussbaum: 1992:222).

Podríamos establecer las relaciones uno a uno de los dos niveles de la teoría, sin embargo, lo que he querido subrayar al realizar esta reconstrucción inicial completa de las capacidades básicas es el siguiente argumento. Nussbaum sostiene una concepción esencialista de la vida humana tal es que cataloga y clasifica una serie de capacidades y límites por los que se puede evaluar. Así mismo, deriva de esa caracterización inicial una serie igual de capacidades que constituyen un umbral mínimo de funcionamiento humano. A partir de allí, Nussbaum sugiere que es posible establecer una concepción de la vida buena con arreglo a estas capacidades que deben ser promovidas por todos los Estados que se preocupen por el bienestar y el respeto a la dignidad humana. Adicionalmente, hay una suerte de independencia en cada una de ellas tal es la carencia de una de ellas, sin importan que se posean ampliamente alguna de las otras, ya es elemento suficiente para calificar una deshumanización de la

${ }^{5} 1$. Being able to live to the end of a complete human life, as far as is possible; not dying prematurely or before one's life is so reduced as to be not worth living.

2. Being able to have good health; to be adequately nourished; to have adequate shelter; having opportunities for sexual satisfaction; being able to move from place to place.

3. Being able to avoid unnecessary and non-beneficial pain and to have pleasurable experiences.

4. Being able to use the five senses; being able to imagine, to think, and to reason.

5. Being able to have attachments to things and persons outside ourselves; to love those who love and care for us, to grieve at their absence, in general, to love, grieve, to feel longing and gratitude.

6. Being able to form a conception of the good and to engage in critical reflection about the planning of one's own life.

7. Being able to live for and with others, to recognize and show concern for other human beings, to engage in various forms of familial and social interaction.

8. Being able to live with concern for and in relation to animals, plants, and the world of nature.

9. Being able to laugh, to play, to enjoy recreational activities.

10. Being able to live one's on life and nobody else's; being able to live one's own life in one's very own surroundings and context (Nussbaum: 1992:222). 
persona. Finalmente, algunas de estas capacidades se tornan arquitectónicas de la vida humana; mientras compartimos necesidades comunes con los animales, la razón práctica y la sociabilidad son las que mantienen unido el conjunto que consideramos humano.

Nussbaum es consiente que esta lista inicial requiere una defensa y una compromiso con un esencialismo. Al respecto señala varios elementos: el primero es que es una lista abierta; sujeta las variaciones culturales que cada región o cultura requiera adecuar. El segundo es su fundamentación histórica y empírica; se aleja de toda concepción metafísica de la persona humana. tercero está sujeta al acuerdo mediante un consenso traslapado; siguiendo este concepto Rawlsiana, Nussbaum va a afirmar que su lista será objeto de un acuerdo político y no metafísico que busca establecer un mínimo acuerdo sobre la concepción humana y sus necesidades tales que podamos emprender un proyecto político para la promoción del justicia y la vida humana digna. Cuarto, este modelo esencialista es fundamentalmente aristotélico y pretende mantener una pluralidad en al menos dos modos diferentes: un modo de especificación plural y uno local. La especificación plural, como su nombre lo indica, implica la planeación Política con una concepción del bien muy general procurando abarcar la totalidad de las concepciones de vida buena. Por si parte el local atiende a las diferencias puntuales de cada cultura bajo el la idea aristotélica de la necesidad de considerar un tipo diferente de especificación plural del bien. Como se puede intuir, es natural que por ejemplo un modo de fomentar la educación en una parte del mundo resulte totalmente ineficaz en la otra (Cf Nussbaum: 1992:223-225).

Una última crítica a la que se anticipa Nussbaum y que vale la pena recuperar es la acusación que hace el liberalismo al descuido de la autonomía si se aceptase un esencialismo internista de este tipo. Para algunas concepciones liberales, esto implicaría socavar la posibilidad que tienen los ciudadanos para elegir una concepción de vida buena. A este respecto Nussbaum señala cuatro elementos: el primero es afirmar que la lista está es de capacidades, no se funciones reales, lo cual deja un amplio espacio de elección para los ciudadanos. No se pretende entonces empujar a los gobiernos a promover 
ciertos valores y acciones sino que busca que los ciudadanos tengan los recursos y condiciones necesarias para desarrollarse en la manera que consideren más acorde con sus nociones de realización. En este sentido entonces, el gobierno debería estimular la elección, no limitarla o eliminarla (Cf Nussbaum: 1992:225).

En segundo lugar, vemos que la elección juega un papel central, -arquitectónico dirá Nussbaum- en la lista. La capacidad para elegir es bien ponderada al interior de su concepción esencialista como elemento constitutivo de toda vida humana. En tercer lugar, el referente político del liberalismo es para Nussbaum John Rawls, y de acuerdo con su perspectiva, no parece repudiar un esencialismo de este tipo pues insiste en que las satisfacciones de un demasiado aumento de en la posibilidad de elección no tienen ningún valor moral tal es que las partes en la posición original gozan de lo que denomina dos poderes morales que ejercen una suerte de restricción sobre el resultado que el acuerdo puede alcanzar. Finalmente, la concepción esencialista aristotélica que pretende defender, no funciona en la espontaneidad; requiere unas condiciones materiales y sociales básicas para su puesta en marcha, por lo que si a alguien le importa la autonomía, debe importarle también el proveer las condiciones materiales para su realización. Así, esta concepción aboga por el florecimiento humano en todas las áreas que la componen y no simplemente en un sesgado conjunto arbitrariamente escogido.

\section{Capacidades básicas y dignidad}

Nussbaum considera pertinente establecer una lista de capacidades básicas que se ajusten a las personas y que le permitan establecer un umbral de medición a fin de determinar si a la persona se le está respetando un mínimo vital de dignidad tal es que su vida valga la pena ser vivida. Una capacidad básica es una facultad innata de la persona que posibilita en última instancia su desarrollo (Cf Nussbaum: 2006:53). Pero para saber qué se necesita para que una vida esté a la altura de la dignidad humana se requiere establecer un mínimo esencial de dignidad que se supone cobija las capacidades básicas. Nussbaum establece un total de 10 capacidades básicas vinculadas a cada 
una de las esferas que componen la vida humana. "1. Vida, 2. Salud física, 3. Integridad fisica, 4. Sentidos, imaginación y pensamiento, 5. Emociones, 6. Razón práctica, 7. Afiliación, 8. Otras especies, 9. Juego, 10. Control sobre el propio entorno, a) Político. b) Material" (Nussbaum: 2006:45-47).

Ahora bien, vimos que el lenguaje del enfoque se subrayaba la capacidad "poder ser o hacer", haciendo alusión principalmente a los derechos, por lo que superficialmente el lenguaje de los deberes está ausente. No obstante, podemos suponer que el lenguaje en el que se establecen las capacidades va acompañado de un requerimiento de protección que implica un deber. $\mathrm{Si}$ alguien reclama un derecho, entonces alguien más está en el deber de proveérselo, y dado que todos podemos exigir para nosotros esas capacidades básicas, ello implica que debemos respetar y promover las de los demás.

La libertad presentada en la Carta Magna puede ser interpretada de diferentes maneras. Si revisamos las diferentes nociones sobre la libertad y su papel en el hilo conductor de los derechos, quizás encontremos un punto desde el cual podamos compararla con la libertad expresada en las capacidades. En los dos últimos siglos podemos distinguir al menos tres grandes ideas de libertad: la libertad como ausencia de interferencia o libertad negativa, la libertad como participación política y la libertad como liberación de la miseria y el hambre.

La libertad como ausencia de impedimentos se remite a lo Constant llamaba "libertad de los modernos" y que luego fue rebautizada por Berlin como libertad negativa. Sostiene que el hombre necesita una esfera privada en la cual pueda hacer uso de su libertad sin interferencias de ningún tipo. Esta noción exige un mínimo de libertad personal como requisito para el desarrollo de las facultades naturales, sociales y políticas. Desde la lógica que acompaña la libertad negativa, se defiende el derecho al libre desarrollo de la personalidad, la libertad de culto, el derecho a la libre expresión y opinión. Esta noción de libertad fue acogida en la declaración francesa de los derechos señalándola como la posibilidad de hacer todo aquello que desee siempre que no perjudique a los demás. 
La libertad como participación, que en la obra de Constant se entendería como la libertad de los antiguos, hace referencia a una noción de libertad como participación en las decisiones políticas que afectan la vida en comunidad. No basta con establecer una zona libre de interferencias para mi desarrollo privado, también considero importante participar activamente en la conformación del poder general, la distribución de los bienes públicos y el destino de la nación. Esta perspectiva de la libertad es acogida dentro de los derechos como libertad para participar en la arena pública y derecho a elegir y ser elegido como representante de una colectividad, entre otras.

Finalmente, la idea de libertad como liberación de la miseria toma como punto de partidas las necesidades de las personas y las posibilidades reales que tienen para satisfacerlas. Esta libertad se expresa en términos de poder ser o hacer, pero ese poder se mide en la capacidad real que tengo para alcanzar los medios que me permitan satisfacer mis necesidades. Aquí podemos establecer una comparación entre los tres sentidos de libertad desde una perspectiva de la legalidad:

Según el primer sentido, soy libre si no existen obstáculos externos que impidan realizar mis objetivos vitales; de acuerdo con el segundo, la no-interferencia resulta insuficiente, puesto que para quien se encuentra en una condición extrema, no significa gran cosa la idea de que no exista impedimentos legales que obstaculicen su acceso a determinados bienes, o que impidan expresamente su conversión en un propietario. [...] La libertad significa [en el tercer sentido] la posibilidad real de satisfacer las necesidades básicas, y la liberación del individuo de aquella forma de esclavitud en la que se transforma de hecho la carencia de los medios vitales mínimos y la lucha diaria por la subsistencia (Papachini, 2003:60-61).

La problemática que gira en torno a las dos primeras concepciones es que su aplicación práctica puede desconocer un factor importante: que no basta legislar la protección de la propiedad, la promoción y defensa de la dignidad si a la par de ello no se establecen unos mecanismos que evalúen y promociones los medios para satisfacer las urgencias diarias de quienes padecen grandes miserias. 
Ahora bien, podríamos preguntarnos ¿A qué idea e libertad hace referencia los documentos sobre los derechos humanos y a cuál el enfoque de las capacidades? Si analizamos la forma en que se incorporan los derechos a las constituciones en los países democráticos, vemos una clara alusión a la protección de los derechos en el sentido de una promoción de la libertad negativa. Como consecuencia, aparecemos libres e iguales ante la ley pero vivimos en condiciones de total desigualdad en bienestar, oportunidades y recursos.

La noción de igualdad por su parte, tanto en la fundamentación de los derechos como en las capacidades, permanecería vigente en sentido muy similar. Las concepciones de persona humana en las dos posturas sugieren que en lo fundamental somos iguales y que es posible establecer parámetros de vida comunes a todos los hombres. No obstante, queda por establecer si esto sí es posible, ya que por ejemplo Sen se ha abstenido de establecer lista de capacidades precisamente porque considera que no se puede universalizar las ideas de vida buena sin que con ello se atropelle nociones culturales y cosmológicas de algunas comunidades. Además, Nussbaum va a reevaluar algunas limitaciones del concepto de persona en los derechos humanos, particularmente en derechos estereotipados y su descuido para con los discapacitados.

En suma, la noción de dignidad es un punto en común entre las capacidades básicas y los derechos. La lista de capacidades va encaminada a lograr y perfeccionar la dignidad humana por lo que existe entre ellas una relación intrínseca. Sin embargo, mientras en muchos casos los derechos tienen como base la dignidad solamente como eje axiomático, las capacidades se encaminan a buscar una vida acorde con ella en la realidad práctica. Esta búsqueda de una vida acorde con la dignidad humana puede ser una herramienta para la labor que la Corte Constitucional viene realizando y que a veces resulta un trabajo muy laborioso que termina por actuar, no en función de una justicia imparcial sino de una concepción abstracta de la dignidad que termina, no por ser autoevidente como cree Kant, sino más bien un chivo expiatorio, o en otras ocasiones una causa de discordia que termina por ser resuelta en base a las preconcepciones del magistrado que las profiera. 


\section{Bibliografía}

Corte Constitucional Sentencia 1084 de 2005. (27) días de octubre de 2005. M.P. Alfredo Beltrán

, Sentencia T-881, 17 de octubre de 2002.

M.P. Eduardo Montealegre

Sentencia T-395 del 3 de agosto de 1998, M.P. Alejandro Martínez Caballero, , Sentencia T-389 del 17 de abril de 2001 , Sentencia T-576 del 16 de julio de 2003,

M.P. Alfredo Beltrán Sierra , Sentencia T-701 del 22 de agosto de 2006, M.P. Álvaro Tafur Galvis. , Sentencia C-397 del 24 de mayo de 2006, M.P. Jaime Araújo Rentería. , Sentencia T-970/14, 15 de diciembre de 2014. M.P. Luis Ernesto Vargas

Constitución Política de Colombia, (1991). Actualizada con los Actos Legislativos hasta 2010. Consejo Superior de la Judicatura, Centro de Documentación Judicial (CENDOJ). Edición digital.

Illie, A. P. (2004). Una aproximación al concepto de dignidad humana. Universitas: Revista de filosofia, derecho y política, (1), 9-13.

KANT, I. (1994). La metafísica de las costumbre. (2a. ed.). MADRID: Tecnos

Leslei H. (2011) The Jurisprudence of Dignity. En: University of Pennsylvania Law Review. Vol. 160, No. 1, pp. 169-233

NACIONES UNIDAS (1945): Carta de Naciones Unidas [En línea]: http://www.cinu.org.mx/onu/documentos/cartatxt.htm

NACIONES UNIDAS (1948): Declaración Universal de Derechos Humanos [En línea]: http://www.un.org/es/documents/ udhr/ 
NACIONES UNIDAS (2003): El Desarrollo Basado en un Enfoque de los Derechos Humanos: Hacia una Comprensión Colectiva entre las Agencias de las Naciones Unidas. Edición Digital

Nussbaum M. (1992). Human Functioning and Social Justice: In Defense of Aristotelian Essentialism. Political Theory [serial online]. 20(2):202. Available from: Academic Search Premier, Ipswich, MA.

Nussbaum, M. \& Sen A. (1993). The Quality of Life. Oxford, Clarenton Press.

(2006). Fronteras de la Justicia, consideraciones sobre la exclusión. Barcelona: Paidos.

(2011). Creating Capabilities, the Human Development Approach. New York, Harvard University Press.

Peces-Barba Martínez, G. (2007). La dignidad humana. Madrid, Dykinson.

Ramelli, A. (2004). Sistema de fuentes del Derecho Internacional Público y bloque de constitucionalidad en Colombia. Cuestiones Constitucionales, 157-175.

Restrepo, A. M. (2011). Acercamiento conceptual a la dignidad humana y su uso en la Corte Constitucional colombiana. En: Revista Electrónica Diálogos de Derecho y Política, Número 6,(2). Pp.1-19.

Papachini, A. (2003) Filosofia y Derechos Humanos. Cali: Universidad del Valle ed.

Tribunal de Justicia de las Comunidades Europeas, Conclusiones del Abogado General Christine Stix-Hackl en el asunto. C-36/02, Omega/Oberbürgermeisterin der Bundesstadt Bonn, presentadas el 18 de marzo de 2004, Párrafo 76 


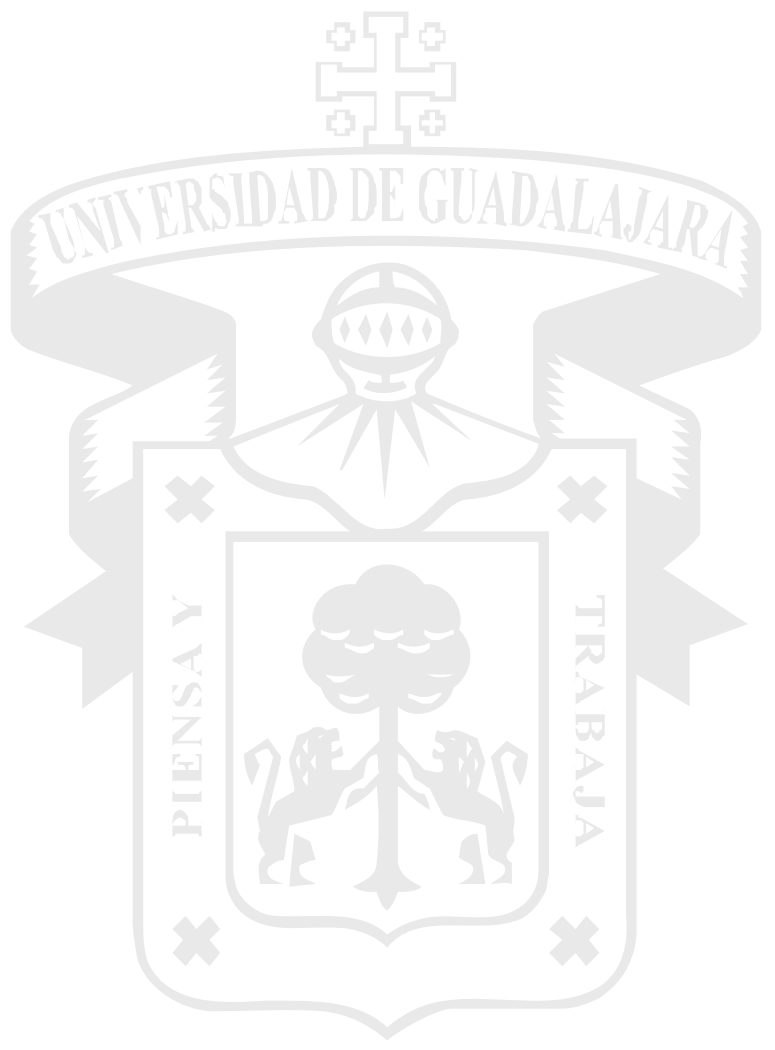

